\title{
A Continuum Model for Current Distribution in Rutherford Cables
}

\author{
Alexander Akhmetov, Luca Bottura, Marco Breschi
}

\begin{abstract}
An analysis of eddy currents induced in flat Rutherford-type cables by external time dependent magnetic fields has been performed. The induced currents generate in turn a secondary magnetic field which has a longitudinal periodicity (periodic pattern). The dependence of the amplitude of the pattern on the history of the cable excitation has been investigated. The study has been carried on with two different models for the simulation of current distribution in Rutherford cables, namely a network model, based on a lumped parameters circuit, and a «continuum» model, based on a distributed parameters circuit. We show the results of simulations of the current distribution in the inner cable of a short LHC dipole model in different powering conditions and compare them to experimental data.
\end{abstract}

Index Terms-Current distribution, Magnetic field pattern, Rutherford cables

\section{INTRODUCTION}

$\mathrm{R}$ UTHERFORD cables used to wind superconducting magnets for particle accelerators are made of a number of non-insulated strands. These strands are twisted and compressed to form a two-layer cable with nearly rectangular cross-section. The time dependent magnetic field that is generated during current ramps can cause long range eddy currents in the superconducting cable, leading to a non uniform current distribution among the strands. In turn, a nonuniformity in the current distribution can cause the premature quench of the magnet resulting in a so-called ramp rate limitation [1]. The study of current distribution can therefore be useful for stability considerations.

Moreover, a 'secondary' magnetic field varying in time and space is associated with the eddy currents. This field component can affect the magnetic field in the dipole bore both directly and indirectly via the magnetization of the superconducting filaments in the strands [2].

In order to investigate these phenomena, an extensive measurement of the dependence of the amplitude of the magnetic field pattern on the powering history of the magnet has been performed in the short LHC dipole model

Manuscript received September 17, 2000

A. Akhmetov is with CERN, LHC Division, Geneva, Switzerland (telephone ++41-22-767-1766, e-mail: alexandre.akhmetov@cern.ch).

L. Bottura is with CERN, LHC Division, Geneva, Switzerland (telephone: ++41-22-767-3729, e-mail: luca.bottura@cern.ch).

M. Breschi is with Università di Bologna, Italy, (telephone: $++39-051$ 2093589, e-mail: marco.breschi@ mail.ing.unibo.it).
MBSMT1. The experimental details and main results are reported in [3]. In this paper we perform a qualitative analysis of the experimental results using two different models to study the current distribution in Rutherford cables.

\section{DESCRIPTION OF THE MODELS AND BASIC ASSUMPTIONS}

We postulate that the periodic magnetic field pattern is due to long current loops circulating in the cable [4,5]. There are at least five reasons for the origination of these currents: nonhomogeneous strand critical properties [4], differences in splice resistances [6] or in the interstrand resistance within the cable [7], strand transposition errors with respect to the background magnetic field, and non-uniform magnetic flux changes along the cable.

In this study we consider a single cable subjected to both a current increase and, proportional to it, a non uniform time dependent magnetic field. The iron saturation at high magnetic fields is neglected. The strands of the cable are assumed to be identical, ideally transposed, and soldered to current leads via electrical resistances which are much lower than the contact resistances of the cable. Finally, the latter are assumed to be equal, and uniform along the cable length.

Hence the only source of long range current loops that we consider here is the field variation along the cable length.

In this study we use two different models. The first one is based on a network approach which approximates the cable as a lumped parameters circuit. The network model is described in detail in [8]. The strands of the upper face are assumed to have contacts with the strands of the lower face but not between themselves. Elementary loops composed of two adjacent strands of the upper face crossing two adjacent strands of the lower face are the main elements of the model. The cable response on the time dependent magnetic flux density $B$ can be studied applying Faraday's and Kirchoff's equations to all loops.

The second model is based on a continuum representation of the cable using a distributed parameters circuit. The model assumes that the current transfer between different strands happens along the length of the cable in a continuous manner and that the voltages induced in the loops of the strands by the variations of the external field are also smeared along the cable length. These driving voltages are proportional to the area enclosed between the strand and the cable center line and to the local value of $\mathrm{d} B / \mathrm{dt}$ directed perpendicular to the cable. A more detailed description of the continuum model is given in [9] and [10]. 


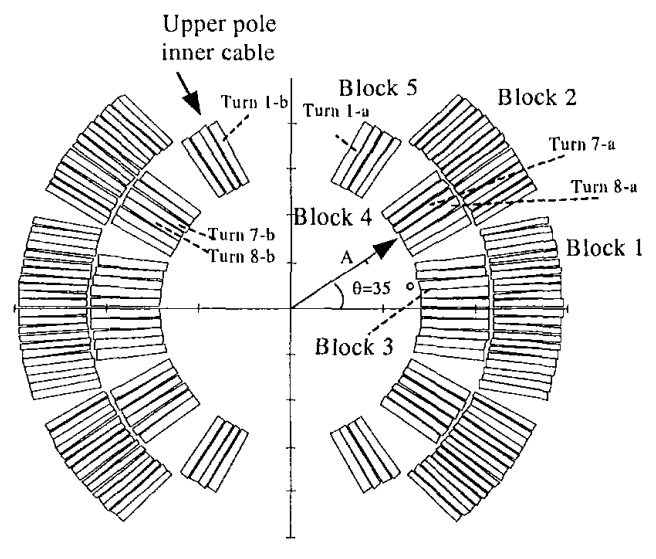

Fig. 1 Cross section of one aperture of the double aperture dipole model MBSMT1 used in the experiments.

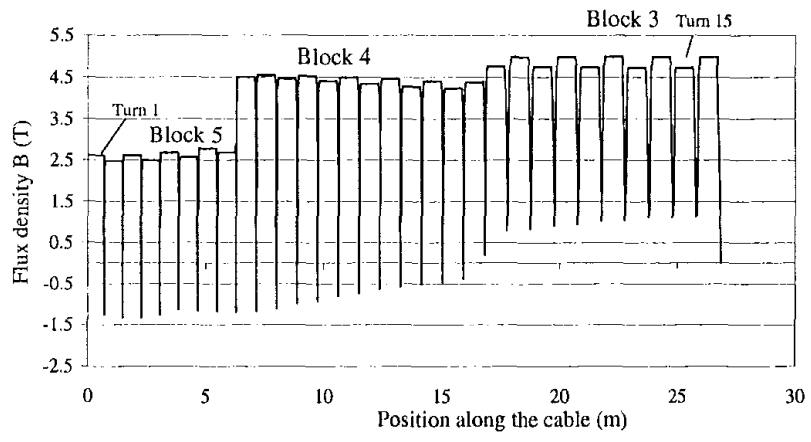

Fig. 2. Magnetic flux density perpendicular to the broad face of the inner cable of a $1 \mathrm{~m}$ long LHC dipole magnet. The reported field is the average field across the cable width calculated with ROXIE, at a total current of $11500 \mathrm{~A}$.

\section{RESULTS AND DISCUSSION}

A schematic view of the coil cross section of one aperture of the LHC dipole model MBSMT1 is shown in Fig. 1. The coil has two poles (upper and lower) assembled from an inner and an outer layer. The inner layer of a single pole is wound from 15 turns arranged into 3 blocks, while the outer layer has 26 turns arranged in 2 blocks corresponding to different angular positions. We have numbered turns and blocks as shown in Fig. 1.

The layers in a pole are wound individually, and the cable ends are either soldered together at the interconnection between layers and poles or connected through splices to the current leads. Because of the assumption of low splice resistance compared to the interstrand resistance in the cable, we can identify a cable unit length in a single layer (i.e. between two splices) as the shortest length to be used for analysis purposes.

In order to simulate the experimental results on the amplitude of the magnetic field pattern reported in [3], we assume that the longitudinal oscillations of the secondary field (the periodic pattern) are only due to the current distribution in the inner cable of the magnet. This assumption is motivated by the fact that the periodicity of the measured oscillations of the secondary magnetic field is equal to the inner cable twist pitch $[3,11]$.

A unit length of the inner layer cable is made of 28 strands, with a total length $L$ of $27.8 \mathrm{~m}$, a twist pitch of $115 \mathrm{~mm}$., and a thickness of $1.88 \mathrm{~mm}$.

As we stated previously, we take spatial variations of the magnetic flux as the only source of long range current loops. The distribution of the magnetic flux density perpendicular to the broad face of the cable calculated with the computer program ROXIE [12] is shown in Fig. 2. We recognize two types of variations of the perpendicular field along the cable length. Sharp, short range variations of the field are due to the cable bending over the magnet bore, while step changes in the value of the field along the straight part of the cable correspond to the transition from block to block.

We have performed two sets of simulations using either the network or the continuum model, in order to imitate the cable excitation with current cycles made of a linear ramp up followed by a current plateau as were performed in the experiments reported in [3].

Simulations with the network model are aimed to look at the fine details of the origination of the short and long range eddy currents. The size of the network model grows quickly with the length of cable analyzed. For this reason we limited the simulations to a short length of cable to explore general features of the transient response of the long range current loops to field changes.

We have then performed simulations of the whole cable response to current steps with the continuum model. The continuum model does not reproduce accurately the details, but is suitable for the simulation of a full cable length in a magnet.

In both sets of simulations we have varied parametrically the flat top current $\left(I_{F T}\right)$ and the ramp rate $(R R)$, in order to study their influence on the current distribution.

\section{A. Network model}

The simulations using the network model have been focussed at an ideal cable close to the coil midplane. In order to minimize the computation time, a four strand cable has been considered. The cable length $L$ is equal to approximately 23 twist pitches. We have assumed a symmetric magnetic field distribution along half of the cable length, shown in Fig. 3. The magnetic field is zero at the cable ends and at its center. In between, there are regions of uniform magnetic field. The magnetic field is twice as high at the inner edge of the cable as at the outer one. This geometry imitates a complete turn in a dipole magnet, where the uniform field regions represent the straight length of the magnet included between the low field regions in the magnet ends.

The model neglects mutual inductances of the strands. The self inductance of the strand on a length equal to its diameter is $L_{\mathrm{i}}$. The results obtained scale then with $L_{\mathrm{i}} / R_{\mathrm{c}}$, where $R_{\mathrm{c}}$ is the contact resistance.

Let us consider elemental current cycles made of a ramp up followed by a current plateau. Let also the plateau current be much less than the critical current. The results of simulations 


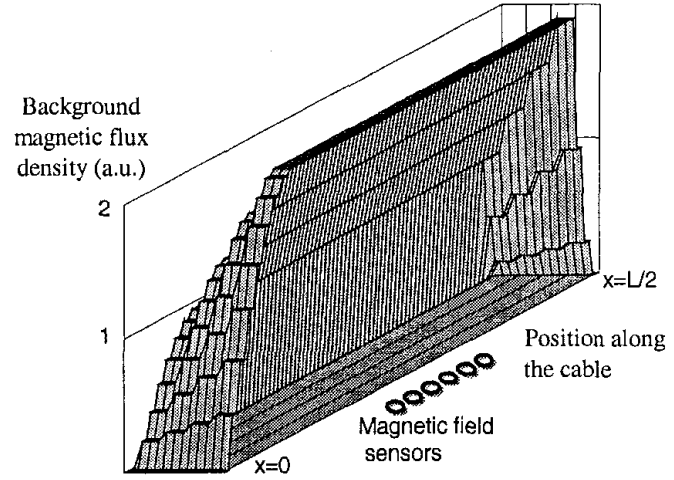

Fig 3 Magnetic flux density perpendicular to the broad face of the ideal cable considered for the simulations performed with the network model.

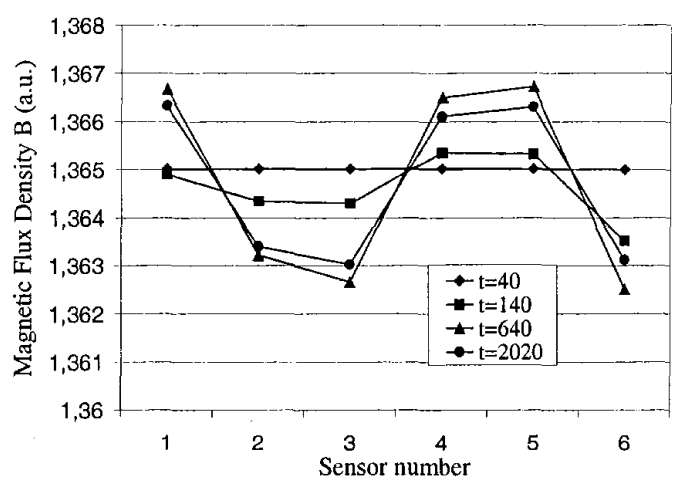

Fig. 4. Development of magnetic field pattern in the uniform field region

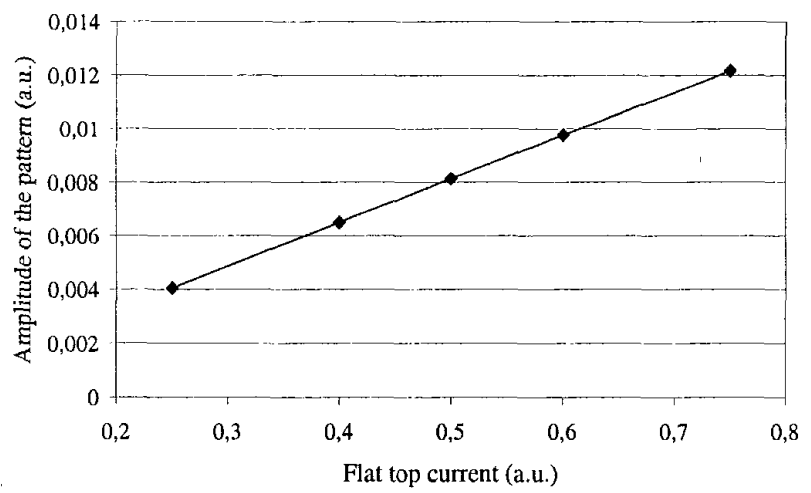

Fig. 5 Dependence of the amplitude of the magnetic field pattern on the flat top current at the end of the ramp.

show that soon after the beginning of the cycle eddy currents associated with the local value of $\mathrm{d} B / \mathrm{dt}$ appear. These eddy currents produce a uniform field in the neighborhood of the cable, without any periodic oscillation.

Gradually, however, the strand currents become different in the ends of the cable, where the magnetic field has a longitudinal gradient, thus originating current loops.

As time advances, the current in these loops diffuses from the cable ends regions into the center. The time $t_{p}$ needed to complete this process is much higher than the time of development of uniform eddy currents.
The buildup and following decay of a periodic magnetic field pattern is shown in Fig. 4. The amplitude of the pattern increases at $\mathrm{t}<\mathrm{t}_{\mathrm{P}}$ and decreases afterwards.

Calculations show that if the time of the ramp is short compared to $t_{p}$, the amplitude of the periodic magnetic field pattern on the plateau at a certain moment, $t>t_{p}$ does not depend on the ramp rate, while it is linearly dependent on the flat top current (see Fig. 5).

This result is in agreement with the scaling found on the experimental data reported in [3].

\section{B. Continuum model}

We have performed calculations with the continuum model for a cable made of 28 strands, as in reality, and with a cable composed of 8 equivalent strands. The equivalent strands are defined so that the total strand length and cross section is the same as for the 28 strands cable.

In accordance with measured value in the dipole model MBSMT1, we have taken a contact resistances between strands in the range 10 to $20 \mu \Omega$. The cable model includes strand self inductance as well as mutual inductances between neighboring sectors of the strands. For details on the homogeneised interstrand conductivity and on the inductance calculation see [10].

We have used the magnetic field map shown in Fig. 2 as an input for the calculation, scaling the field proportionally to the current in the magnet. In the simulations performed with the continuum model we have neglected the field variations across the cable width. This, as demonstrated in the detailed analysis discussed in the previous section, is inessential for the development of long range current loops.

As we have postulated that the magnetic field pattern is due to long current loops circulating in the cable, it is reasonable to assume that its amplitude is proportional to the difference between the currents flowing in the strands. In particular we have taken the values of the maximum difference between the strand currents in the middle of the uniform field regions, $\Delta I_{\text {max }}$, as the reference quantity for a qualitative comparison with the amplitude of the magnetic field pattern measured outside the cable and next to these positions.

As an example we compare in Fig. 6 the amplitude of the total magnetic field pattern at position $\mathrm{A}(r=17 \mathrm{~mm}$ and $\theta=$ $35^{\circ}$ ) to the parameter $\Delta I_{\max }$ calculated for turn 7-a which faces the point selected. The amplitude of the total field pattern has been reconstructed at point $\mathrm{A}$ from the data presented in [3], obtained with the rotating coils placed in the middle of the straight length of the magnet. The comparison is shown for a step response measurement with $I_{F T}=2000 \mathrm{~A}$ and $R R=50$ $\mathrm{A} / \mathrm{s}$. We have performed the simulations with the complete 28 strands cable, for different values of the cross contact resistances (10 and $20 \mu \Omega$ ).

After few seconds from the ramp start strong differences between the strand currents start propagating from the regions of non-uniform field towards the straight parts of the cable, thus creating the periodic oscillations of the magnetic field outside the cable. During the flat top both field oscillations and non homogeneities of current distribution start to decay. 


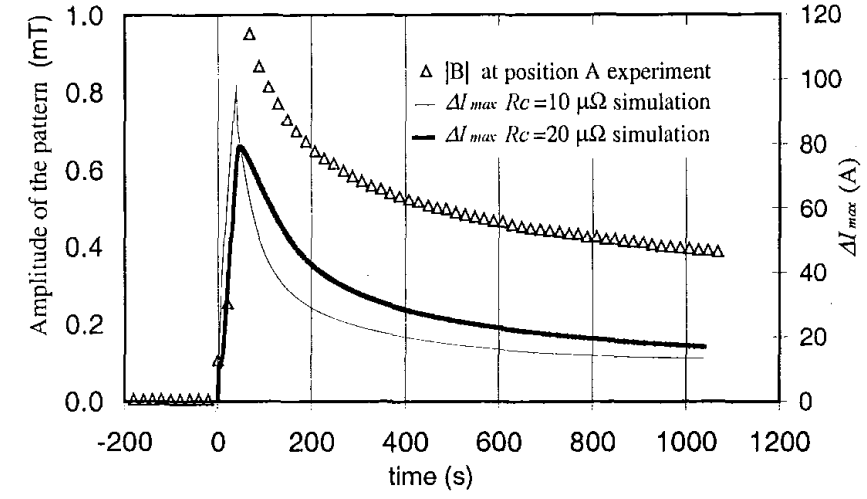

Fig. 6 Time evolution of the amplitude of the periodic pattern in the middle of the uniform field region and of parameter $\Delta I_{\max }$ calculated at turn 7-a (step response with $I_{F T}=2000 \mathrm{~A}, R R=50 \mathrm{~A} / \mathrm{s}$ ). Time $\mathrm{t}$ is set to zero at the beginning of the current ramp.

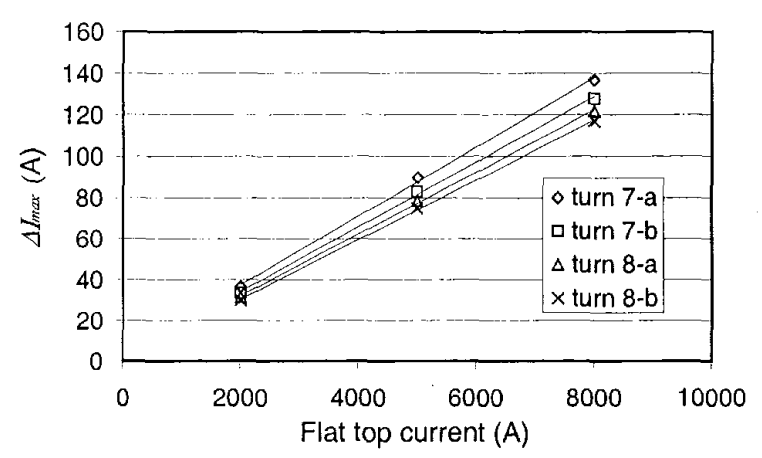

Fig. 7. Dependence of parameter $\Delta l_{\max }$ on the final flat top current in different cable turns $(R R=450 \mathrm{~A} / \mathrm{s})$.

The value of $\Delta I_{\max }$ at the end of the ramp calculated in four consecutive straight parts of the cable (turns 7-8, see Fig. 1) is shown in Fig. 7 for the case of $R R=450 \mathrm{~A} / \mathrm{s}$. These simulations have been performed with the equivalent, 8 strands cable for the whole set of ramp rates and flat top currents considered in the experiments, and the results confirm that the amplitude of $\Delta I_{\max }$ at the end of the ramp is proportional to the flat top current and approximately independent on the ramp rate, as obtained from the network model and in the experiments [3].

Calculations also reveal that during the ramp the current distribution in consecutive turns of the cable is quite similar. In this phase the sharp, short range variations of the magnetic field in the ends induce large current peaks with a periodic structure, and a period equal to half a turn.

At longer times these peaks diffuse and a coherent current difference builds-up in the cable over the complete length. We attribute these residual and large long range currents to the field variations associated to the transition from block to block.

\section{CONCLUSIONS}

Long range eddy current loops generated by a non-uniform and time dependent magnetic field cause a periodic variation of the field in accelerator magnets wound from Rutherford cables. We have used two different models of current distribution in Rutherford cables to analyze the dependence of these eddy currents on the powering history of the cable.

The two models have a different range of application. The network model gives fine details on the cable currents, but is limited by a rapidly growing computational size. On the other hand the continuum model does not focus on fine details, but allows to study real cables made of some tens of strands along the whole cable length.

Both models confirm that the amplitude of the magnetic field periodic pattern at the end of the ramp of a simple current cycle made of a ramp-up followed by a plateau is proportional to the plateau current and, for short ramps, independent on the ramp rate. This result is in good agreement with the experimental findings reported in [3].

\section{ACKNOWLEDGMENT}

We gratefully acknowledge Dr. Stephan Russenshuck from CERN, LHC Division, for having provided the field maps for the calculations. We also wish to acknowledge Prof. Pierluigi Ribani from the University of Bologna, Department of Electrical Engineering, for many fruitful discussions.

\section{REFERENCES}

[1] Schermer RI. "Status of Superconducting Magnets for the Superconducting Super Collider», IEEE Trans Mag.., 30, 1587, 1994

[2] R. Wolf, «The Decay of the Field Integral in Superconducting Accelerator Magnets wound with Rutherford Cables", Presented MT15, Beijing, China, 1997.

[3] L. Bottura, M. Breschi, M. Schneider, «Measurements of Magnetic Field Pattern in a Short LHC Dipole Models, to appear in IEEE Trans. Appl. Sup.

[4] Akhmetov, A. Devred, R. Mints, R. Schermer, «Current Loop Decay in Rutherford-Type Cables», Supercollider-5, Plenum Press, N.Y., 443, 1994.

[5] L. Krempasky, L. Schmidt, «Influence of a longitudinal variation of $\mathrm{dB} / \mathrm{dt}$ on the magnetic field distribution of superconducting accelerator magnets», Appl. Phys. Lett., 66, 1545, (1995).

[6] N. Mitchell, «Analysis of non-uniform current distribution effects in multistage cable-in-conduit conductors», Cryogenics, vol 40 (2), pp. 99-116, 2000.

[7] A.P. Verweij, «Electrodynamics of Superconducting cables it Accelerator Magnets», Ph.D. Thesis, University of Twente, Enschede, (NL), 1995.

[8] A. Akhmetov, A. Devred, T. Ogitsu, «Periodicity of Cross-over Currents in a Rutherford-type Cable Subjected to a Time Dependent Magnetic Field», Jour. Appl. Phys, vol. 75 (6), pp. 3176-3183, 1994.

[9] L. Bottura, M. Breschi, F. Negrini, P. L. Ribani, «Electromagnetic analysis of current distribution in multistrand superconducting cables», Inst. Phys. Conf. Ser., 167, 1191-1194, 2000.

[10] A. Akhmetov, L. Bottura, M. Breschi, P. L. Ribani, «Theoretical Analysis of Current Imbalance in Flat Two Layer Superconducting Cables», presented at CHATS Y2K, Frascati, Italy, September 2000.

[11] A. K. Ghosh, K. E. Robins, W. B. Sampson, «The Ramp Rate Dependence of the Sextupole Field in Superconducting Dipoles», IEEE Trans. Mag., vol. 30 (4), pp. 1718-1721, 1994.

[12] Procs, of the First Int. ROXIE users meeting, S. Russenschuck ed., Cern Report 99-01, Geneva, 1999. 